\title{
Optimum design of brake friction material using hybrid entropy-GRA approach
}

\author{
Naresh Kumar ${ }^{1}$, Tej Singh ${ }^{1, a}$, R.S. Rajoria ${ }^{2}$, and Amar Patnaik ${ }^{3}$ \\ ${ }^{1}$ Department of Mechanical Engineering, Manav Bharti University, Solan-173229, India \\ ${ }^{2}$ Department of Mechanical Engineering, CEC Landran-140307, India \\ ${ }^{3}$ Department of Mechanical Engineering, M.N.I.T. Jaipur-302017, India
}

\begin{abstract}
The effect of Kevlar and natural fibres on the performance of brake friction materials was evaluated. Four friction material specimens were developed by varying the proportion of Kevlar and natural fibres. Two developed composite contained 5-10 wt.\% of Kevlar fibre while in the other two the Kevlar fibre was replaced with same amount of natural fibre. SAE J661 protocol was used for the assessment of the tribological properties on a Chase testing machine. Result shows that the specimens containing Kevlar fibres shows higher friction and wear performance, whereas Kevlar replacement with natural fibre resulted in improved fade, recovery and friction fluctuations. Further hybrid entropy-GRA (grey relation analysis) approach was applied to select the optimal friction materials using various performance defining attributes (PDA) including friction, wear, fade, recovery, friction fluctuations and cost. The friction materials with $10 \mathrm{wt} \%$ of natural fibre exhibited the best overall quality.
\end{abstract}

\section{Introduction}

Generally, a friction material contains a resin binder, fibrous reinforcement, abrasives, fillers and lubricants in varying composition [1]. Asbestos because of its economical issue and met the bulk of fascinating properties of brake friction material are used for years as a fibrous reinforcement [2]. However, the ban on the utilization of asbestos by environmental protection agencies because of its carcinogenic nature had forced the friction industry to hunt alternative of asbestos [3]. Last two decades witnessed a lot of research in the development of asbestos-free brake friction materials [410]. In this way the formulation designers start focusing on natural fibres and industrial wastes as a source of raw materials for friction formulations [11-17]. The utilization of such materials will not only be economically viable, but may also result in reduced environmental pollution. A good friction material requires an appropriate set of performance defining attributes (PDAs) namely high and stable coefficient of friction, negligible fading, faster recovery, better wear resistance and low-sensitivity towards load-speed alterations for reliable and safe performance [1]. Therefore assessment of such PDAs and selection of optimal friction material can be regarded as multiple criteria decision-making (MCDM) problem [18-20]. Hence in this work the comparative effect of addition of Kevlar and natural fibre on the tribo-performance of brake friction material is studied and optimal formulation is selected by using MCDM approach.

\footnotetext{
${ }^{\mathrm{a}}$ Corresponding author: tejschauhan@gmail.ocom
}

\section{Experimental}

\subsection{Materials and fabrication of friction specimens}

Friction materials based on phenolic resin, Kevlar fibre, Lapinus fibre, graphite, barite, alumina, vermiculite, potassium titanate and natural fibre amounting to $100 \%$ by weight were fabricated as per Table 1 . Detail of the processing conditions for composite fabrication is briefly reported elsewhere [21-23].

Table 1. Composition and nomenclature of the friction materials specimens.

\begin{tabular}{lllll}
\hline \multirow{2}{*}{ Composition (wt.\%) } & \multicolumn{4}{l}{ Nomenclature } \\
\cline { 2 - 5 } & $\mathrm{K}-1$ & $\mathrm{~K}-2$ & $\mathrm{~N}-1$ & $\mathrm{~N}-2$ \\
\hline *Parent composition & 45 & 45 & 45 & 45 \\
Barite & 50 & 45 & 50 & 45 \\
Kevlar Fibre & 5 & 10 & 0 & 0 \\
Natural Fibre & 0 & 0 & 5 & 10 \\
\hline
\end{tabular}

K: Kevlar fibre, N: Natural fibre

*Parent composition: Phenolic resin, Lapinus fibers, Alumina, Graphite, Potassium Titanate and Vermiculite.

\subsection{Tribo-performance evaluation methodology}

The tribo-performance of the specimens was evaluated on a chase type friction material testing machine following SAE J661a standard. The details of the testing protocol and testing machine is reported elsewhere [24]. The performance coefficient friction, fade, recovery, wear, 
friction fluctuations and cost were considered as PDA of the evaluation. The detailed description of the various PDAs used in this study is given as:

PDA-1: $\mu$-Performance (Higher-is-better)

It is the average friction coefficient of all fade and recovery cycles.

PDA-2: Wear (Lower-is-better)

It is the loss of material from the specimen during braking. It is calculated as, $\Delta W=W_{1}-W_{2}$, where $W_{1}$ the weight of specimen before test and $W_{2}$ is the weight of the specimen after test.

PDA-3: \%-Fade (Lower-is-better)

$\%$-Fade $=\frac{\mu_{\text {Performance }}-\mu_{F}}{\mu_{\text {Performance }}} \times 100$, where $\mu_{\mathrm{F}}$ is the minimum coefficient of friction for the fade cycles. Lower \%-fade is desirable for good friction composites. The standard range is $0-30 \%$.

PDA-4: \%-Recovery (Higher-is-better)

$\%$-Recovery $=\frac{\mu_{\mathrm{R}}}{\mu_{\text {Performance }}} \times 100$, where $\mu_{\mathrm{R}}$ is the maximum coefficient of friction for the recovery cycles. It is desirable in the range of $100-140 \%$ for good friction composites.

PDA-5: Frictional fluctuations (Lower-is-better)

Frictional fluctuations are given by $\Delta \mu=\mu_{\max }-\mu_{\min }$. Where, $\mu_{\max }$ is the highest coefficient of friction and $\mu_{\min }$ is the lowest coefficient of friction.

PDA-6: Cost (Lower-is-better)

It is the cost of the composition per kilogram.

\subsection{Ranking evaluation methodology}

The ranking evaluation methodology consists of three main phases as:

Phase I: Identification of the alternatives, PDAs and decision matrix

Phase II: Weight determination of various PDAs

Phase III: Ranking of the alternatives

Phase I: Identification of the alternatives and PDAs

Here the number of alternatives and performance defining attributes (PDAs) are identified. After the identification of alternatives and criterions the values of experimental results for each alternative is expressed in a decision matrix as:

$$
\begin{aligned}
& \begin{array}{llll}
C_{1} & C_{j} & \cdots & C_{n}
\end{array} \\
& X=\begin{array}{c}
A_{1} \\
A_{i} \\
\vdots \\
A_{m}
\end{array}\left[\begin{array}{llll}
x_{11} & x_{1 j} & \cdots & x_{1 n} \\
x_{i 1} & x_{i j} & \cdots & x_{i n} \\
\vdots & \vdots & \ddots & \vdots \\
x_{m 1} & x_{m j} & \cdots & x_{m n}
\end{array}\right]
\end{aligned}
$$

where, $A_{1}, \ldots, A_{m}$ are the composition/alternatives and $C_{1}, \ldots, C_{n}$ are the PDA. Hence, $x_{i j}$ is the value of $\mathrm{j}^{\text {th }}$ PDA $\left(C_{j}\right)$ for the $\mathrm{i}^{\text {th }}$ composition/alternative $\left(A_{i}\right)$.

Phase II: Entropy method for weight determination
Entropy method is used for the calculation of weight. This method consists of the following steps [25]:

Step I: The constructed matrix is normalized using following equation:

$$
N_{i j}=\frac{x_{i j}}{\sum_{i=1}^{m} x_{i j}}
$$

Step II: In this step, the entropy for each PDA is calculated as:

$$
e_{j}=-\kappa \sum_{j=1}^{n} N_{i j} \ln \left(N_{i j}\right)
$$

Where, $\kappa$ is a constant and calculated as $\kappa=\frac{1}{\ln (m)}$

Step III: Finally the weight of each PDA is calculated as:

$$
\varpi_{j}=\frac{\partial_{j}}{\sum_{j=1}^{n} \partial_{j}}
$$

Where, $\partial_{j}=1-e_{j}$ and it is the degree of diversity of the information involved in the outcomes of the $\mathrm{j}^{\text {th }}$ PDA.

Phase III: Ranking of the alternatives

Grey system theory was developed by Deng and consists following step [26]:

Step-I: In order the make the values free of unit the normalization process is done. The normalized values of each element in the performance matrix can be calculated as follows:

$$
\begin{aligned}
& X_{i j}^{\prime}=\frac{x_{i j}-\min \left\{x_{i j}\right\}}{\max \left\{x_{i j}\right\}-\min \left\{x_{i j}\right\}} \\
& X_{i j}^{\prime}=\frac{\max \left\{x_{i j}\right\}-x_{i j}}{\max \left\{x_{i j}\right\}-\min \left\{x_{i j}\right\}}
\end{aligned}
$$

Where $\max \left\{x_{i j}\right\}$ and $\min \left\{x_{i j}\right\}$ respectively are the maximum and minimum values of the $i^{\text {th }}$ PDA. Eq. (5) is used for the larger-the-better PDA; Eq. (6) is used for the smaller-the-better PDA.

Step-II: In this step reference sequence $\left(\mathfrak{R}_{j}\right)$ is generated from the normalized matrix by taking the largest normalized value of each PDA as:

$\mathfrak{R}_{j}=\max _{i=1}^{m}\left\{X_{i j}^{\prime}\right\}$

Step-III: Grey correlation coefficient for each term is determined as:

$$
\Phi_{j}=\frac{\min _{i=1}^{m} \min _{i=1}^{n} \Delta_{i j}+\hbar \max _{i=1}^{m} \max _{i=1}^{n} \Delta_{i j}}{\Delta_{i j}+\hbar \max _{i=1}^{m} \max _{i=1}^{n} \Delta_{i j}}
$$

Where $\Delta_{i j}=\left|\Re_{j}-X_{i j}^{\prime}\right|$ and $\hbar$ is known as the distinguishing coefficient and generally takes the value of 0.5 .

Step-IV: Finally a grey relational degree (which is the weighted sum of the grey relational coefficients) is calculated by the following equation: 
$\Omega_{i}=\sum_{j=1}^{n}\left[\Phi_{j} \times \varpi_{j}\right]$

Where $\varpi_{j}$ is the weight of the $\mathrm{j}^{\text {th }}$ PDA and calculated using entropy method. Therefore, using the grey correlation degrees the friction material formulation sequence can be prioritized and the one with the highest degree of correlation will be identified as the best formulation that represents the optimal solution.

\section{Results and discussions}

\subsection{Experiment results}

The tribo-performance evaluation of the fabricated friction composites materials were carried out on a chase type testing machine. The experiment results corresponding to each PDA are listed in Table 2. The performance friction coefficient ( $\left.\mu_{\text {Performance }}\right)$ $(0.586 \pm 0.009)$ and wear performance $(1.065 \pm 0.055 \mathrm{~g})$ remains higher for Kevlar filled friction composite. The $\mu_{\text {Performance }}$ and wear performance are observed to decrease for the friction composition having natural fibre content. From fade performance point of view natural fibres $(30.39 \% \pm 5.96)$ based friction composition proved much superior to Kevlar fibre based composition where fade remains highest $(54.91 \% \pm 8.52)$. The recovery performance is observed to exceed $100 \%$ irrespective of the fibrous reinforcement. It remains $\sim 110.435 \pm 1.525 \%$ for Kevlar based composition and increases slightly to $110.735 \pm 1.765 \%$ for the compositions having natural fibre as reinforcement. Moreover, natural fibres based compositions performed better from friction fluctuations point of view and are much cost effective as compared to Kevlar fibre based compositions.

\subsection{Ranking of the alternatives}

The decision matrix from Eq.1 is used for the entropyGRA analysis. The weights of the selected PDAs are calculated by using Eqs. 2-4 and given in Table 3. The weight of PDAs as evaluated by entropy method are PDA-1 $=0.009, \quad$ PDA-2 $=0.078, \quad$ PDA-3 $=0.205, \quad$ PDA$4=0.003$, PDA $-5=0.185$, PDA- $6=0.520$. After weight calculation, GRA technique is applied to find the optimum composition using Eqs. 5-9. The GRA technique results are shown in Table 6. From this analysis, it was observed that the natural fibre based compositions exhibits the optimal performance.

Table 2. Experimental data of the PDAs.

\begin{tabular}{lllllll}
\hline Alternatives & PDA-1 & PDA-2 & PDA-3 & PDA-4 & PDA-5 & PDA-6 \\
\hline K-1 & 0.595 & 1.12 & 46.39 & 108.91 & 0.329 & 105.25 \\
K-2 & 0.577 & 1.01 & 63.43 & 111.96 & 0.435 & 169.75 \\
N-1 & 0.520 & 1.70 & 36.35 & 112.50 & 0.262 & 45.25 \\
N-2 & 0.524 & 1.20 & 24.43 & 108.97 & 0.175 & 49.75 \\
\hline
\end{tabular}

Table 3. Weight of PDAs as calculated by entropy method.

\begin{tabular}{lllllll}
\hline Alternatives & PDA-1 & PDA-2 & PDA-3 & PDA-4 & PDA-5 & PDA-6 \\
\hline Entropy & 0.998 & 0.984 & 0.959 & 0.999 & 0.963 & 0.895 \\
Weight & 0.009 & 0.078 & 0.205 & 0.003 & 0.185 & 0.520 \\
\hline
\end{tabular}

Table 4 The normalized decision matrix and reference sequence.

\begin{tabular}{lllllll}
\hline Alternatives & PDA-1 & PDA-2 & PDA-3 & PDA-4 & PDA-5 & PDA-6 \\
\hline K-1 & 1.000 & 0.841 & 0.437 & 0.000 & 0.408 & 0.518 \\
K-2 & 0.760 & 1.000 & 0.000 & 0.850 & 0.000 & 0.000 \\
N-1 & 0.000 & 0.000 & 0.694 & 1.000 & 0.665 & 1.000 \\
N-2 & 0.053 & 0.725 & 1.000 & 0.017 & 1.000 & 0.964 \\
& 1 & 1 & 1 & 1 & 1 & 1 \\
Reference sequence & 1 & & & & \\
\hline
\end{tabular}

Table 5 Grey relation coefficient.

\begin{tabular}{lllllll}
\hline Alternatives & PDA-1 & PDA-2 & PDA-3 & PDA-4 & PDA-5 & PDA-6 \\
\hline K-1 & 1.000 & 0.759 & 0.470 & 0.333 & 0.458 & 0.509 \\
K-2 & 0.676 & 1.000 & 0.333 & 0.769 & 0.333 & 0.333 \\
N-1 & 0.333 & 0.333 & 0.620 & 1.000 & 0.599 & 1.000 \\
N-2 & 0.342 & 0.642 & 1.000 & 0.333 & 1.000 & 0.932 \\
\hline
\end{tabular}


Table 6 Grey correlation degree and ranking of the formulations.

\begin{tabular}{llllllllc}
\hline Alternatives & PDA-1 & PDA-2 & PDA-3 & PDA-4 & PDA-5 & PDA-6 & Grey correlation degree & Ranking \\
\hline K-1 & 0.009 & 0.059 & 0.096 & 0.001 & 0.085 & 0.265 & 0.515 & 3 \\
K-2 & 0.006 & 0.078 & 0.068 & 0.002 & 0.062 & 0.173 & 0.389 & 4 \\
N-1 & 0.003 & 0.026 & 0.127 & 0.003 & 0.111 & 0.52 & 0.790 & 2 \\
N-2 & 0.003 & 0.050 & 0.205 & 0.001 & 0.185 & 0.485 & 0.929 & 1 \\
\hline
\end{tabular}

\section{Conclusions}

Optimal design of friction material using combined entropy-GRA approach was carried out in this work. The optimal design of friction material was performed in three phases: (1) selection of alternatives and performance defining attributes, (2) weight calculation using entropy method and (3) GRA method is used to rank the alternatives. In this study, Kevlar and natural fibre based friction materials were selected as alternatives whereas; performance coefficient of friction, wear, fade, recovery, frictional fluctuations and cost were selected as performance defining attributes. Based on entropy-GRA results, the ranking of the friction materials from the best to the worst is in the order of $\mathrm{N}-2, \mathrm{~N}-1, \mathrm{~K}-1$, and $\mathrm{K}-2$. The specimen $\mathrm{N}-2$ having $10 \mathrm{wt} . \%$ of natural fibre was ranked best among all the evaluated friction materials.

\section{References}

1. J. Bijwe, Polymer Composites 18, 378 (1997)

2. D. Chan, G.W. Stachowiak, Proc. Instn Mech. Engrs- Part D: J. Automobile Engineering 218, 953 (2004)

3. T. Singh. Ph.D. Thesis, N.I.T. Hamirpur (2013)

4. B.K. Satapathy, J. Bijwe, Wear 257, 573 (2004)

5. X. Qu, L. Zhang, H. Ding, G. Liu, Polymer Composites 25, 94 (2004)

6. T. Singh, A. Patnaik, Archives of Civil and Mechanical Engineering 15, 151 (2015)

7. T. Singh, A. Patnaik, R. Chauhan, A. Rishiraj, Journal of King Saud University: Engineering Sciences. DOI: 10.1016/j.jksues.2015.06.002, (2015)

8. T. Singh, A. Patnaik, B.K. Satapathy, American Institute of Physics Conference proceeding 1393, $223(2011)$

9. T. Singh, A. Patnaik, B.K. Satapathy, American Institute of Physics Conference proceeding 1536, 259 (2013)

10. T. Singh, A. Patnaik, B. Gangil, Journal of Industrial Textile; DOI: 10.1177/1528083714559568, (2014)

11. N. Dadkar, B.S. Tomar, B.K. Satapathy, A. Patnaik, Materials and Design 31, 723 (2010)

12. A. Tiwari, H.S. Jaggi, R.K. Kachhap, B.K. Satapathy, S.N. Maiti, B.S. Tomar, Wear 309, 259 (2014)

13. T. Singh, A. Patnaik, R. Chauhan, Materials and Design 89, 1335 (2016)

14. A. Mustafa, M.F.B. Abdollah, F.F. Shuhimi, N. Ismail, H. Amiruddin, N. Umehara, Materials and Design 67, 577 (2015)
15. U.D. Idris, V.S. Aigbodion, I.J. Abubakar, C.I. Nwoye, Journal of King Saud UniversityEngineering Sciences 27, 185 (2015)

16. K.K. Ikpambese, D.T. Gundu, L.T. Tuleun, Journal of King Saud University-Engineering Sciences, http://dx.doi.org/10.1016/j.jksues.2014.02.001, (2014).

17. D.S. Yawas, S.Y. Aku, S.G., Amaren, Journal of King Saud University-Engineering Sciences, http://dx.doi.org/10.1016/j.jksues.2013.11.002, (2013)

18. T. Singh, A. Patnaik, B. Gangil, R. Chauhan, Wear 324-325, 10 (2015)

19. T. Singh, A. Patnaik, B.K. Satapathy, M. Kumar, Composites: Mechanics, Computations, Applications. An International Journal 3, 189 (2012)

20. T. Singh, A. Patnaik, B. K. Satapathy, Walailak Journal of Science and Technology 10, 343 (2013)

21. T. Singh, A. Patnaik, B.K. Satapathy, M. Kumar, B.S. Tomar, Journal of Materials Engineering and Performance 22, 796 (2013)

22. T. Singh, A. Patnaik, B.K. Satapathy, NANO 8, 1 (2013)

23. T. Singh, A. Patnaik, Polymer Composites: DOI:10.1002/pc.23682 (2015).

24. P. Gopal, L.R. Dharani, F.D. Blum, Wear 193, 199 (1996)

25. T.C. Wang, H.D. Lee, Expert Systems with Applications 36, 8980 (2009)

26. J.L. Deng, Systems and Control Letters 1, 288 (1982) 